ISSN 1983-8484

Licenciado sob uma Licença Creative Commons

\title{
Importância da mensuração de desempenho industrial na indústria: um estudo de caso
}

\author{
Importance of industrial performance measurement in industry: a case study
}

Teonas Bartz ${ }^{[a]}$, Julio Cezar Mairesse Siluk ${ }^{[\mathrm{b}]}$, Luís Eduardo Barth ${ }^{[\mathrm{c}]}$

[a] Mestrando em Engenharia de Produção pelo Programa de Pós-Graduação em Engenharia de Produção da Universidade Federal de Santa Maria (UFSM), Santa Maria, RS - Brasil, e-mail: efficacy.consultoria@gmail.com

[b] Doutor em Engenharia de Produção pela Universidade Federal de Santa Catarina (UFSC), professor da Universidade Federal de Santa Maria (UFSM), Santa Maria, RS - Brasil, e-mail: jsiluk@ufsm.br

[c] Graduando em Engenharia de Produção pela UNIVATES, Lajeado, RS - Brasil, e-mail: luisbarth@gmail.com

\section{Resumo}

Este artigo apresenta um estudo de caso realizado nos setores produtivos de uma indústria de manufatura de plásticos. O problema encontrado era que a empresa controlava apenas indicadores financeiros do negócio, sendo que não haviam indicadores de desempenho para o setor produtivo. Em decorrência de desconhecer seus índices de desempenho, a empresa não avaliava a causa de problemas comuns e especiais, o que estava aumentando os custos industriais e reduzindo a margem operacional. 0 principal objetivo deste artigo foi desenvolver um método simples de controle de indicadores, os quais auxiliem a tomada de decisão no setor produtivo. Para alcançar esse objetivo, foi realizada uma pesquisa aplicada, de natureza quantitativa, descritiva, desenvolvida por um estudo de caso. Com o conhecimento teórico de alguns modelos de medição de desempenho e do processo, foram quantificadas as principais causas de problemas. Em seguida, foram criados indicadores de desempenho para cada setor e globais, de acordo com as necessidades da empresa. Esses indicadores de desempenho tratam os índices de materiais sucateados, de devoluções internas e externas e de tempo de máquinas paradas. Após um período inicial de coleta de dados, em fontes bibliográficas e de análise documental, se observou que os índices estão, em sua maioria, fora de controle. Isso demonstra a importância de haver controles e metas para os elementos-chave da operação de qualquer organização. Como resultados desta pesquisa, apresentam-se as telas de controle de indicadores geradas por meio de planilhas eletrônicas, onde os gestores visualizam a situação de seus indicadores em comparação com as metas propostas. Concluise que a elaboração deste controle tornará mais rápida a tomada de ações da empresa frente a problemas potenciais, tornando a empresa mais ágil e competitivas.

Palavras-chave: Gestão estratégica. Indicadores de desempenho. Melhoria de processos. Competitividade. 


\section{Abstract}

This article presents a case study in the productive sectors of the plastics manufacturing industry. The problem encountered was that the company controlled only financial indicators of the business, and there were no performance indicators for the productive sector. As a result of not knowing of its performance indexes, the company did not track the cause of common problems and specials, which was increasing manufacturing costs and reducing the operating margin. The main aim of this paper was to develop a simple method of control indicators, which assist decision making in the productive sector. To achieve this goal, we conducted an applied research, quantitative, descriptive, developed by a case study. With the knowledge of some theoretical models of performance measurement and process, quantified as the main causes of problems. After the performance indicators were created for each sector and global, according to business needs. These performance indicators address the contents of scrapped materials, internal and external returns and time machine stops. After an initial period of data collection, bibliographic sources and document analysis, it appears that the contents are mostly out of control. This demonstrates the importance of having controls and targets for the key elements of the operation of any organization. As a result of this research, we present the control screens of indicators generated by spreadsheets, where managers view the status of its indicators in comparison with the proposed objectives. It is concluded that the development of this control will make it quick to action against the company's potential problems, making the company more agile and competitive.

Keywords: Strategic management. Performance indicators. Process improvement. Competitiveness.

\section{Introdução}

De acordo com Suzigan (2001 apud OLIVEIRA et al., 2010), os avanços na medição de desempenho ocorreram mais acentuadamente na mensuração de indicadores econômicos. A controladoria cresceu e tornou esta área bastante presente nas organizações. Porém, na área industrial ainda existem melhorias a serem realizadas, através de pesquisas, para auxiliar no diagnóstico e gestão das áreas de manufatura, logística, qualidade, comercial e outras.

Para Teles e Vartanian (1997 apud LOZECKY, 2004), "mensurar é atribuir números a propriedades de um determinado objeto ou evento devidamente especificado". Porém, cabe salientar que um sistema de mensuração de desempenho precisa ser consistente e adequado. Corrêa e Hourneaux Junior (2008), baseados em Harrington (1993), apresentam as principais mudanças apresentadas na mensuração com o passar dos anos, visto que a gestão organizacional evoluiu e cada vez mais as informações são necessárias para gerir as empresas. Essa evolução pode ser observada no Quadro 1.

Pode-se observar que os modelos iniciais de mensuração de desempenho eram baseados, basicamente, na análise financeira. As limitações de modelos de gerenciamento apenas com medidas financeiras eram grandes, mas as primeiras tentativas de mudar os sistemas de controle foram sem medidas de inter-relacionamento entre setores e sem relações estratégicas. Uma das limitações, no entanto, é que as empresas pensam que o sistema de medição de desempenho é o responsável pelo sucesso na obtenção de resultados. Esquecem que a vantagem dos indicadores de desempenho está na qualidade da estratégia escolhida e a capacidade de implementá-la para interpretar e gerenciar os dados obtidos. A importância inicial da mensuração de desempenho é conhecer uma situação a qual está se querendo controlar mais profundamente. Nas organizações este conhecimento é importante para saber em que situação se encontram os diferentes setores, fornecendo dados para a tomada de decisão.

Kaplan e Norton (1997) enfatizam o que grandes gurus da qualidade já pregavam na época de explosão da implantação de programas de qualidade, afirmando que é muito importante medir os resultados da empresa, pois sem esta avaliação não há como gerenciar as atividades. Assim, a mensuração de resultados de diferentes setores é de extrema importância para que as organizações consigam avaliar, comparar e corrigir o seu desempenho (CORRÊA; HOURNEAUX JUNIOR, 2008).

0 objetivo deste trabalho foi desenvolver um sistema de medição de desempenho industrial que fosse de fácil aplicação prática no setor produtivo de uma empresa industrial. Com a criação de indicadores de desempenho adequados, que supram as 
Administração por objetivos - APO

É uma técnica de planejamento estratégico, para médio e longo prazo; e tático, a curto prazo; que trata de objetivos gerais para o longo prazo, que podem ser conhecidos como carta de intenções. Os objetivos gerais são o passo inicial para a implantação desse modelo. Após, definem-se os objetivos setoriais, pelos próprios departamentos envolvidos, a partir do objetivo geral. As metas podem ser individuais, sendo direcionadas a pessoas e derivam dos objetivos setoriais (FERREIRA; PEREIRA; REIS, 2006).

Para aplicar a APO, deve-se realizar o plano para atingir as metas departamentais; um programa de atendimento e atividades específicas para atender as metas; um projeto para avaliar as vantagens e desvantagens e normas de desempenho para cada etapa.

Quadro 1 - Mudanças na mensuração de desempenho

\begin{tabular}{ll}
\hline \multicolumn{1}{c}{ Antes } & \multicolumn{1}{c}{ Depois } \\
\hline Medição dos produtos & Medição de processos e serviços \\
Administração de lucros & Administração de recursos \\
Realização de metas & Aperfeiçoamento contínuo \\
Medições de quantidades & Medições de eficácia, eficiência e de adaptabilidade \\
Medições baseadas em especificações técnicas ou empresariais & $\begin{array}{l}\text { Medições baseadas nas expectativas dos clientes internos e } \\
\text { externos }\end{array}$ \\
Atenção concentrada no indivíduo (indivíduos são capazes de & Atenção concentrada no processo (os processos determinam \\
controlar os resultados) & os resultados e devem ser controlados) \\
Processo imposto de cima para baixo & Equipe desenvolve e gerencia o desempenho \\
\hline
\end{tabular}

Fonte: CORRÊA; HOURNEAUX JUNIOR, 2008.

\section{S's Mckinsey}

0 modelo de medição de desempenho da Mckinsey foi denominado por 7S por compreender que a relação entre sete fatores, onde a letra inicial das palavras originais em inglês é "S". Esse modelo busca obter um sistema de gerenciamento equilibrado, dividindo a organização em partes elementares, através da utilização dos sete fatores. São eles: estratégia, estrutura, sistemas, estilo, equipe de direção, habilidades e valores comuns.

A identificação e obtenção dos fatores considerados como "hard" é facil, encontram-se nos planos estratégicos, diagramas organizacionais e outras do- cumentações. Os quatro fatores considerados "soft" possuem maior dificuldade em sua identificação e consequentemente obtenção, pois capacidades, valores e elementos culturais estão continuamente sendo adquiridos e mudados (CRUZ et al., 2005).

\section{Key performance áreas - KPA}

Para Rentes (2000), as áreas-chave de desempenho são os poucos fatores principais que direcionam o sucesso da organização na realização de suas estratégias e objetivos. Para Brown (2000), os fatores-chave de sucesso ou área-chave de desempenho são 
definidos em três ou cinco áreas gerais da empresa nas quais a organização deve focalizar para alcançar sua visão, identificar medidas de desempenho, estratégias e ajudar a organização a priorizar ações e investimentos. As áreas-chave de desempenho podem ser diferentes tipos de objetos como, por exemplo: processos operacionais; funções específicas da organização; elementos externos, como a percepção do cliente; aspectos infraestruturais, como a estrutura de treinamento existente.

\section{Modelo Baldrige}

O Programa Baldrige é formado por uma parceria público-privada nos Estados Unidos - EUA, dedicada à excelência do desempenho organizacional. Ela está ligada ao Instituto Nacional de Padronização e Tecnologia, agência do Departamento de Comércio americano. O Programa Baldrige educa organizações na gestão de excelência de desempenho e administra o prêmio nacional de qualidade Malcolm Baldrige (GEORGE, 1993).

O modelo Baldrige é um programa de avaliação de desempenho organizacional consagrado internacionalmente. Segundo os critérios de excelência de desempenho para o ciclo 2011-2012, disponíveis no site oficial do prêmio Baldrige, o principal objetivo do programa é melhorar o desempenho das organizações, através de uma estratégia de avaliação. Para alcançar este objetivo principal, o programa trabalha para: aumentar a consciência sobre a importância da excelência do desempenho na condução da economia dos EUA e global; fornecer instrumentos de avaliação organizacional e os critérios; educar os líderes das empresas, escolas, organizações de saúde e agências governamentais e sem fins lucrativos sobre as melhores práticas de gestão; e reconhecer modelos nacionais de gestão e os honrar com o Prêmio Presidencial para a excelência do desempenho (NIST, 2011).

Para avaliar e premiar as organizações, este sistema desenvolveu um processo abrangente de diferentes critérios de avaliação baseados na observação e conhecimentos obtidos através da análise de diversos especialistas de todo os EUA sobre modelos e práticas de gestão. Esta diversidade de conhecimentos e informações influenciou no desenvolvimento de critérios flexíveis e genéricos, os quais poderiam ser aplicados a diversas áreas e setores da sociedade (DAVIS; AQUILANO; CHASE, 2001). Este sistema apresenta modelos de avaliação de desempenho em cinco áreas: saúde, educação, governo, organizações sem fins lucrativos e negócios. Este trabalho focará somente a área relacionada com negócios.

\section{Key performance indicator - KPI}

Para Latorre, Roberts e Riley (2010), os indicadores são medidas de desempenho quantificáveis que auxiliam as empresas a definir, avaliar e melhorar sua performance nas áreas mais importantes para a organização. Além de medir o desempenho da organização, leva a todos os níveis hierárquicos as diretrizes, objetivos e estratégias, envolvendo todos os colaboradores na realização dos objetivos estratégicos da empresa. Como são medidas estratégicas da organização, devem refletir os direcionamentos gerais da mesma. Assim, cada empresa poderá ter indicadores diferentes, baseados em sua necessidade frente ao mercado. Por isso, os indicadores de desempenho visam melhorar continuamente os processos principais para obter vantagem competitiva.

De maneira geral, seria interessante que todas as organizações tivessem de fazer acompanhamentos sobre fatores internos e externos. Os internos dizem respeito aos seus processos, enquanto os externos levam em consideração elementos dos fornecedores e clientes. Normalmente estão presentes medidas de qualidade, custos e prazos de entregas. Mais recentemente, para atender à nova visão estratégica de algumas empresas, elementos de sustentabilidade e meio ambiente começam a aparecer nos indicadores.

Os indicadores-chave de desempenho, também chamados de KPI's, ajudam as organizações a compreender o quão bem eles estão se saindo em relação a suas metas e objetivos estratégicos. No sentido mais amplo, um KPI fornece as informações de desempenho mais importantes que permitem que organizações ou seus agentes visualizem se estão no caminho certo ou não. Com isso, a implantação dos KPI's inicia com a identificação dos objetivos da organização que lhe permitam cumprir sua missão e trazer valor para todos os envolvidos com a organização. Uma vez definidos, passa-se a monitorá-los e medi-los.

\section{Performance Prism}

Esse modelo de medição de desempenho foi desenvolvido por Neely, Adams e kennerley (2002). 
da formulação. Na sequência há as etapas de melhoria, desenvolvimento, revisão e aprendizado.

cujo foco está na criação de valor para as partes interessadas (clientes, fornecedores, parceiros, empregados, comunidade e governo), a partir da construção de indicadores que auxiliem na obtenção da máxima satisfação dessas partes. Além de ser um modelo de medição de desempenho, pode servir como ferramenta de gestão. Sua abordagem consiste em cinco perspectivas de desempenho inter-relacionadas - as quais representam as cinco faces do prisma - que apresentam as questões fundamentais relacionadas à satisfação das partes interessadas, estratégias, processos, capacidades e contribuição dos parceiros. o Performance Prism é um modelo de medição inovador, sendo considerado como um sistema de avaliação da gestão da organização, de segunda geração.

\section{Balanced Scorecard - BSC}

O BSC foi desenvolvido por Kaplan e Norton em 1992, e caracteriza-se entre os mais importantes modelos de gestão da década de 90.0 objetivo do BSC é traduzir a visão e a estratégia das empresas em um conjunto abrangente de medidas de desempenho, que servem de base para um sistema de medição e gestão estratégica (KAPLAN; NORTON, 2004).

0 modelo de gestão baseia-se em quatro perspectivas estratégicas: aprendizado e crescimento; cliente; financeiro e processos internos do negócios. Segundo Kaplan e Norton (2004), para cada uma das perspectivas, devem ser gerados: objetivos estratégicos; indicadores; metas e iniciativas.

\section{Total Performance Scorecard - TPS}

É um modelo sistêmico de gestão, que trata do desenvolvimento pessoal de todos os membros da organização e a melhoria de suas capacidades para a realização do mais alto desempenho da organização. É um processo sistemático, contínuo, gradual e rotineiro de melhoria, desenvolvimento e aprendizado, que se concentra no aprimoramento sustentável do desempenho pessoal e organizacional (CHANG; MORGAN, 2000).

O ciclo do TPS envolve a formulação do Balanced Scorecard Pessoal e do Balanced Scorecard Organizacional seguida pela fase de comunicação e interligação, na qual são analisados em conjunto o resultado

\section{Metodologia}

O desenvolvimento desta pesquisa adotou uma metodologia científica, que pode ser classificada sobre diferentes perspectivas: quanto à utilização é uma pesquisa aplicada, pois utiliza de conhecimentos disponíveis para estudo e aplica-os para a solução de um problema específico de uma empresa. Quanto à natureza da pesquisa, é classificada como quantitativa, pois os dados coletados serão os indicadores de desempenho industrial após a implantação deste modelo de controle de indicadores. Em relação aos objetivos, esta pesquisa é classificada como descritiva, pois tem a finalidade de descrever a relação entre os indicadores de desempenho industrial. Além desta finalidade, descreverá toda a metodologia a ser utilizada para a implantação nos demais setores de produção da empresa estudada. Em relação às técnicas de pesquisa, foi desenvolvido um estudo de caso, que se iniciou pela elaboração de uma revisão bibliográfica sobre alguns modelos de medição de desempenho. Em seguida, escolheu-se o KPI e para cada setor da empresa foram criados indicadores essenciais para o bom andamento das atividades produtivas destes. Após a criação dos indicadores, foram criadas planilhas eletrônicas para lançamento de dados e geração de gráficos de controle em comparação com a meta estabelecida para cada indicador. Os resultados mostram a importância da monitoração do desempenho das atividades para a tomada de decisões e de ações corretivas e preventivas caso o processo mostre-se fora de controle.

\section{Resultados}

A empresa estudada pertence ao ramo da manufatura de produtos plásticos. Está no mercado há mais de vinte anos, mas até então não trabalhava com indicadores de desempenho para o processo produtivo. Por isso, em um primeiro momento foi escolhido o método de medição baseado no KPI, onde a empresa foi dividida em setores e para cada um destes foram determinados os indicadores de desempenho mais importantes para o controle do processo.

Para avaliar o resultado global da unidade industrial foram criados os indicadores de produção total, 
perda técnica, sucata, devoluções e tempo de máquina parada. Para cada indicador foi desenvolvida planilha eletrônica, onde os dados são lançados e automaticamente são gerados gráficos de controle, para acompanhamento dos índices em comparação com as metas estipuladas.

Analisando-se os cinco setores individualmente, além dos índices gerados a partir das informações apontadas pelos relatórios de produção, cada indicador foi acompanhado da análise das causas, através do cálculo da porcentagem de peso de cada causa, em função da produção total da empresa. A Tabela 1 mostra os valores de produção total, perdas técnicas de processo e perdas anormais, ou seja, a sucata que deve ser evitada no processo.

A Figura 1, gerada a partir destes dados, mostra uma tendência de elevação nos índices de apara total, além da média móvel desse indicador.

Observa-se que sem um indicador de medição de desempenho, a empresa poderia avaliar o resultado através de outros índices que não mostrariam a realidade do setor produtivo. Os dados mostram que mesmo no mês onde a produção diminuiu, o índice de aparas totais aumentou. Além disso, realizando-se um benchmarking com empresas do mesmo ramo, determinou-se uma meta de $11 \%$ para o total de aparas. Assim, a empresa pode tomar ações de correção e prevenção sempre que o índice ultrapassar ou estiver próximo da meta.

Além dos resultados internos, a empresa considerou importante quantificar os índices de devoluções internas e externas. As devoluções internas são importantes devido a quantificar o setor que mais necessita de melhorias para atender aos clientes com a qualidade planejada de acordo com suas estratégias. As devoluções externas refletem o nível de qualidade repassado aos clientes, o que prejudica a imagem da empresa. Além disso, o custo industrial da devolução, seja interna ou externa, é o mais alto desde a entrada da matéria-prima, pois além de todas as etapas do processo, todos os custos de transporte são arcados pela empresa.

Tabela 1 - Perdas totais e sucata geral do processo

\begin{tabular}{|c|c|c|c|c|c|c|}
\hline \multicolumn{7}{|c|}{ Análise geral das aparas } \\
\hline & Maio & $\%$ & Junho & $\%$ & Julho & $\%$ \\
\hline Apara total (kg) & 71.229 & 11,45 & 74.813 & 12,64 & 89.613 & 13,91 \\
\hline Sucata (kg) & 19.374 & 3,11 & 24.147 & 4,08 & 26.847 & 4,17 \\
\hline Perdas técnicas $(\mathrm{kg})$ & 51.855 & 8,33 & 50.666 & 8,56 & 62.766 & 9,75 \\
\hline Produção total $(\mathrm{kg})$ & \multicolumn{2}{|c|}{622.219} & \multicolumn{2}{|c|}{591.781} & \multicolumn{2}{|c|}{644.070} \\
\hline
\end{tabular}

Fonte: Dados da pesquisa.

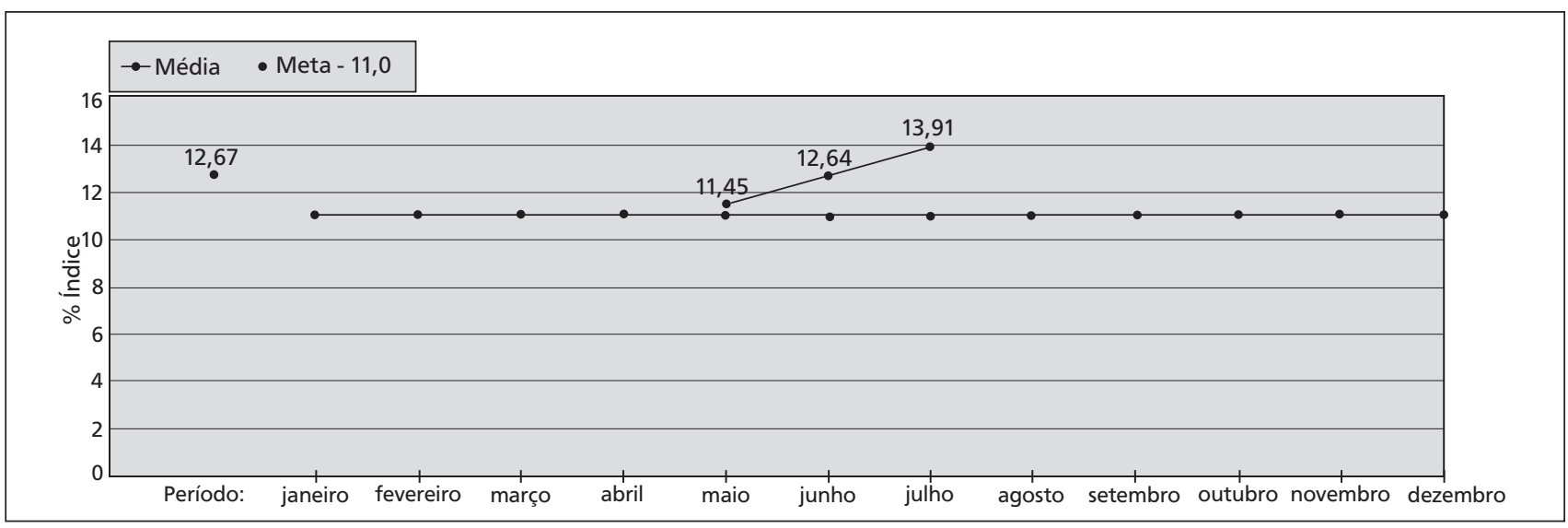

Figura 1 - Produção total e perdas totais de processo

Fonte: Dados da pesquisa. 
Os dados de devolução interna e externa são mostrados na Tabela 2, que relaciona a quantidade devolvida com a produção total da empresa, gerando o índice. A meta definida pela alta direção para este indicador foi de zero, devido ao fato de ser considerado não poder haver devoluções.

Com esses dados foi construída a Figura 2, que plota os valores de devoluções internas e externas em um gráfico de controle. Esse gráfico auxilia na visualização do desempenho do indicador em comparação com a meta estipulada.

Vê-se pelo gráfico que as devoluções estão com índices acumulados de $0,52 \%$ e $0,72 \%$, onde as devoluções internas caíram no mês de julho e as devoluções externas tiveram um pico de 1,3\%.

Nota-se que, após a criação dos indicadores de desempenho, passaram-se três meses de coleta de dados, donde se deduz que a empresa poderia ter um resultado melhor se as causas desses problemas já tivessem sido avaliadas e minimizadas. Já analisando-se os setores individualmente, foram criados indicadores específicos em função da necessidade e da particularidade de cada setor.

Para o setor 1, que representa o início do processo de fabricação da empresa, foram analisadas sete ocorrências de problemas de processo, onde a maior causa foi relativa a acerto de máquinas. Os dados coletados para cada tipo de ocorrência, durante os meses de maio, junho e julho de 2011, são apresentados na Tabela 3.

Considerando os maiores índices de problemas e consequente geração de material a ser sucateado, observa-se, pelos dados da Tabela 3, que as principais causas são o acerto de máquinas e as perdas técnicas. Como as perdas técnicas não podem ser evitadas, controlou-se, inicialmente, o índice de acerto de máquinas através de gráficos. Esse gráfico é representado pela Figura 3.

Tabela 2 - Devoluções internas e externas

\begin{tabular}{|c|c|c|c|c|c|c|}
\hline \multicolumn{7}{|c|}{ Devoluções - 2011} \\
\hline Ocorrências & Maio & $\%$ & Junho & $\%$ & Julho & $\%$ \\
\hline Devoluções internas (kg) & 2.412 & 0,388 & 5.906 & 1,00 & 1.092 & 0,17 \\
\hline Devoluções externas (kg) & 2.666 & 0,428 & 2.534 & 0,43 & 8.354 & 1,297 \\
\hline Produção total (kg) & \multicolumn{2}{|c|}{622.219} & \multicolumn{2}{|c|}{591.781} & \multicolumn{2}{|c|}{644.070} \\
\hline
\end{tabular}

Fonte: Dados da pesquisa.

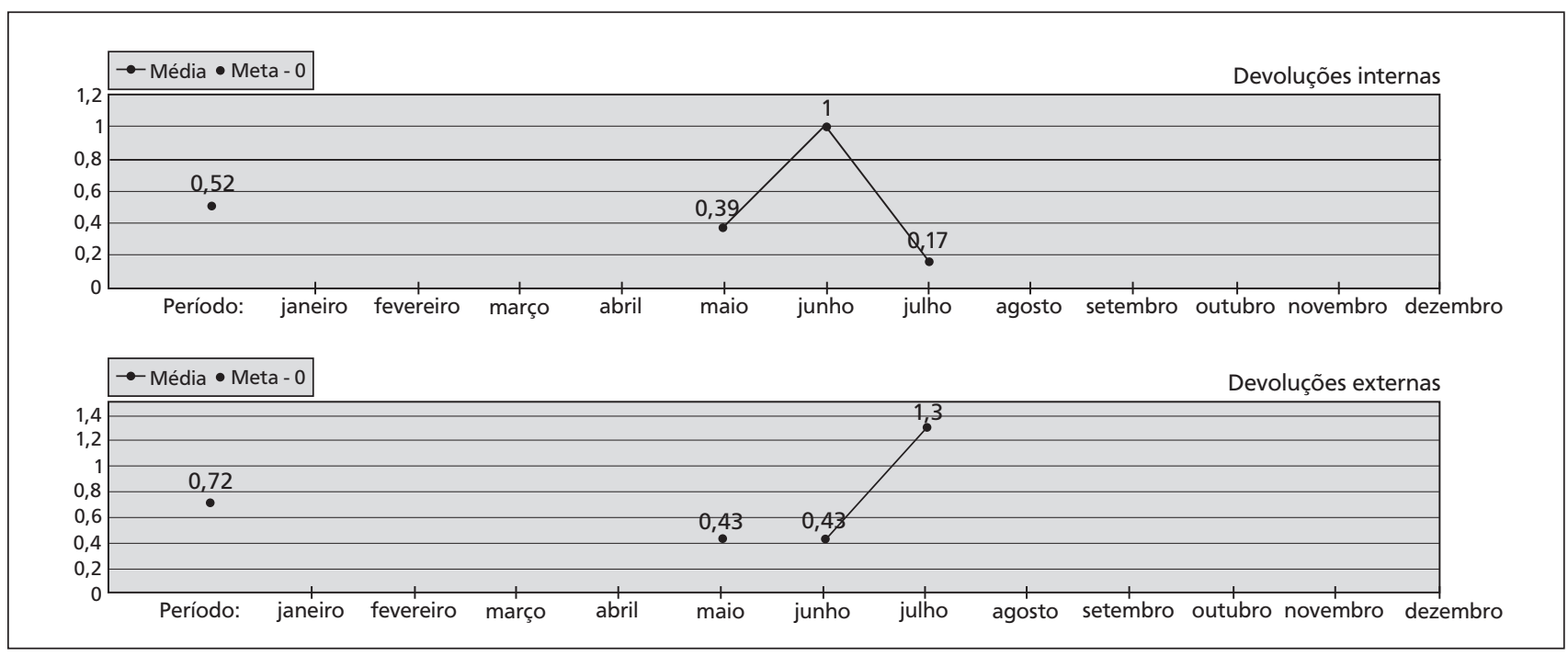

Figura 2 - Devoluções internas e externas

Fonte: Dados da pesquisa. 
BARTZ, T.; SILUK, J. C. M.; BARTH, L. E.

Tabela 3 - Principais problemas no setor 1

\begin{tabular}{|c|c|c|c|c|c|c|}
\hline \multicolumn{7}{|c|}{ Análise das aparas (kg) } \\
\hline Ocorrências & Maio & $\%$ & Junho & $\%$ & Julho & $\%$ \\
\hline Problema operacional (kg) & 463 & 0,10 & 542 & 0,12 & 612 & 0,135 \\
\hline Acerto de máquinas $(\mathrm{kg})$ & 4.657 & 0,97 & 5.082 & 1,10 & 4.871 & 1,071 \\
\hline Problema mecânico (kg) & 510 & 0,11 & 596 & 0,13 & 563 & 0,124 \\
\hline Problema com material $(\mathrm{kg})$ & 0 & 0 & 123 & 0,03 & 30 & 0,007 \\
\hline Perda técnica(kg) & 3.506 & 0,73 & 2.415 & 0,52 & 1.979 & 0,435 \\
\hline Varredura (kg) & 367 & 0,08 & 482 & 0,10 & 326 & 0,072 \\
\hline Caroço (kg) & 399 & 0,08 & 287 & 0,06 & 464 & 0,102 \\
\hline Produção total (kg) & \multicolumn{2}{|c|}{478.085} & \multicolumn{2}{|c|}{462.891} & \multicolumn{2}{|c|}{454.878} \\
\hline
\end{tabular}

Fonte: Dados da pesquisa.

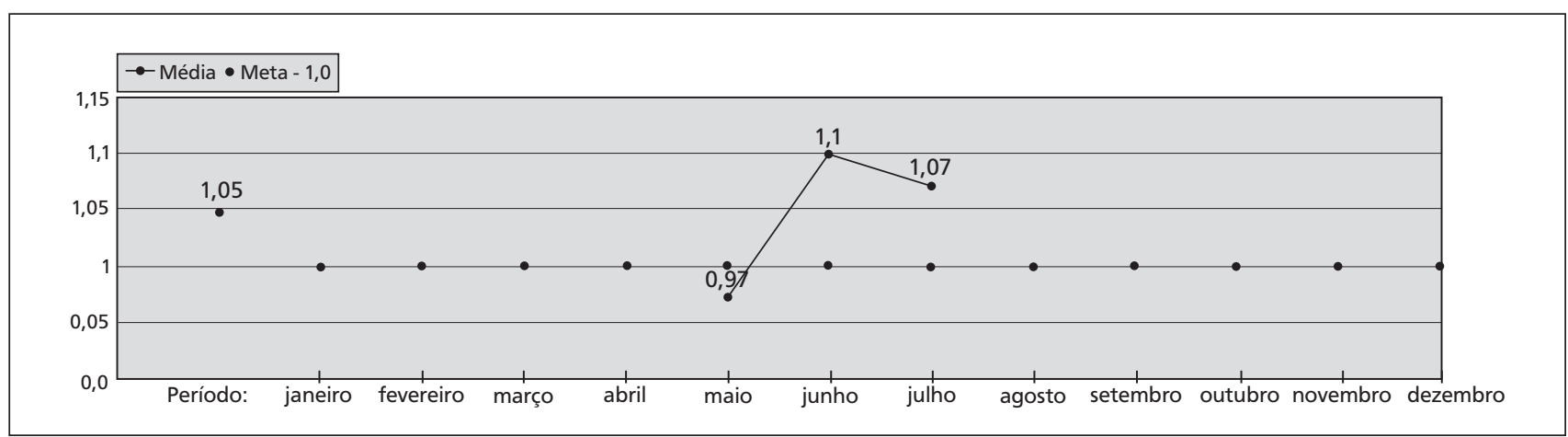

Figura 3 - Indicador de desempenho de acerto de máquinas no setor 1

Fonte: Dados da pesquisa.

Observa-se pelo gráfico que o acerto de máquinas apresenta uma variação média acima da meta determinada, de $1 \%$. Isso pode ocorrer em função do tamanho dos pedidos (kg/pedido) ter diminuído ou por problemas gerados na preparação ou durante o processo, causando a necessidade de acerto dos equipamentos para produção de outro produto, para que o equipamento não permaneça parado.

0 setor 2 representa o maior investimento da empresa, tanto em preparação para produção quanto em tecnologia. Nesse setor, a meta estabelecida através de benchmarking e de históricos anteriores foi de 1,4\% para bobinas de acerto, por esse ser o maior custo da produção. Independente do tamanho dos lotes, o tempo de ajuste e preparação é o mesmo. A Tabela 4 mostra os dados coletados nesse setor produtivo.

Inicialmente foi construído gráfico de controle e acompanhamento para a ocorrência de sucata provocada pelas bobinas de acerto, onde se vê que houve aumento no índice, passando de 1,02\% no primeiro mês analisado para $1,59 \%$ nos dois últimos meses. A média acumulada, 1,4\%, está acima da meta determinada. Essa variação pode ser observada na Figura 4.

Observa-se pela Tabela 4 que os principais problemas relacionam-se com a preparação dos equipamentos para produção. Por isso, além do indicador de desempenho, foi criado o lote mínimo de produção. Considerando que os equipamentos são de grande porte e altos custos operacionais, qualquer período parado representa grandes perdas à empresa.

Além desse controle, por ser o setor onde o custo de processar os materiais é o mais alto na empresa, foi realizado controle de retiradas de serviços de máquinas, onde se verificou que muitas vezes os equipamentos ficam parados por problemas operacionais 
simples, que poderiam ser evitados com a implantação de um procedimento operacional padrão, para que todas as pessoas do processo realizem suas tarefas da mesma maneira e analisem os problemas com a mesma metodologia.

A Tabela 5 mostra as principais causas de retirada de serviço de máquina, e em consequência o tempo que as máquinas permaneceram sem produção, devido a problemas de outros setores ou de preparação no próprio setor 2 .

Observa-se que o índice de tempo de máquina parada aumentou do primeiro mês de controle para o segundo. Pela Tabela 6 vê-se que o número de pedidos retirados de máquina aumentou, assim como a quantidade de materiais sucateados.

Analisando-se as causas, verifica-se pela Tabela 6 que problemas causados pelo próprio setor caíram de 1.095 para 570 minutos e problemas causados por setores que fornecem materiais ou serviços aumentaram significativamente os tempos de paradas de máquina. Por isso, para esse indicador está sendo realizado acompanhamento por meio de gráfico. A meta determinada para este indicador foi de 1,5\% e o gráfico de acompanhamento é visto na Figura 5.

0 setor 3 recebe materiais dos setores 1 e 2 . Ainda, as maiores causas de problemas são sobras de materiais e aparas do processo. As sobras de materiais representam as maiores perdas. Para essa ocorrência foi proposto teto de $1,5 \%$. Os dados são mostrados na Tabela 7.

No setor 3, pode-se verificar que a maior causa de geração de aparas é a sobra de lâmina plástica cortada. Assim, foi determinada meta de 1,5\% sobre a produção do setor para este tipo de ocorrência, sendo que nos três meses avaliados, o índice oscilou acima da meta, o que é verificado no gráfico da Figura 6 .

Tabela 4 - Principais problemas no setor 2

\begin{tabular}{|c|c|c|c|c|c|c|}
\hline \multicolumn{7}{|c|}{ Análise das aparas $(\mathbf{k g})$} \\
\hline Ocorrências & Maio & $\%$ & Junho & $\%$ & Julho & $\%$ \\
\hline Problema com material $(\mathrm{kg})$ & 372 & 0,08 & 370 & 0,10 & 835 & 0,16 \\
\hline Problema operacional (kg) & 649 & 0,14 & 434 & 0,11 & 536 & 0,103 \\
\hline Problema mecânico (kg) & 25 & 0,01 & 189 & 0,05 & 223 & 0,043 \\
\hline Perdas técnicas (kg) & 2.144 & 0,46 & 2.265 & 0,59 & 1.996 & 0,383 \\
\hline Acerto de máquinas (kg) & 1.220 & 0,26 & 1.210 & 0,32 & 1.625 & 0,312 \\
\hline Final de bobina lisa $(\mathrm{kg})$ & 1.745 & 0,37 & 1.678 & 0,44 & 2.549 & 0,49 \\
\hline Bobinas de acerto $(\mathrm{kg})$ & 4.784 & 1,02 & 6.075 & 1,59 & 8.293 & 1,593 \\
\hline Produção (kg) & \multicolumn{2}{|c|}{469.661} & \multicolumn{2}{|c|}{381.664} & \multicolumn{2}{|c|}{520.723} \\
\hline
\end{tabular}

Fonte: Dados da pesquisa.

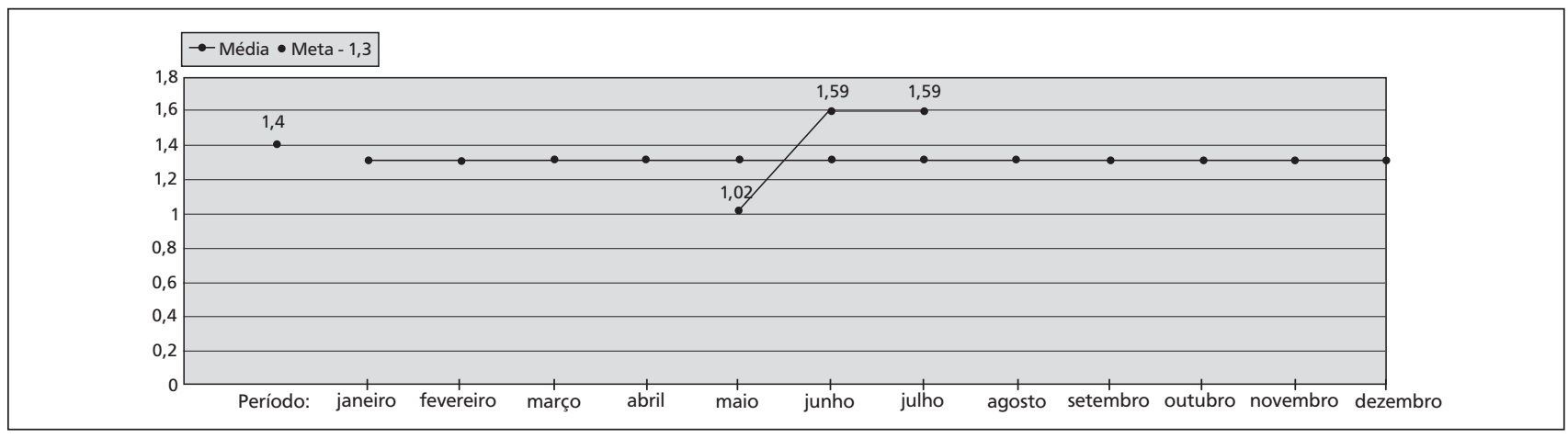

Figura 4 - Indicador de desempenho de sucata por bobinas de acerto no setor 2

Fonte: Dados da pesquisa. 
Tabela 5 - Identificação do setor responsável pela retirada

\begin{tabular}{|c|c|c|c|c|c|c|c|c|c|c|}
\hline \multirow[t]{2}{*}{ Mês } & \multirow{2}{*}{$\begin{array}{r}\text { Colagem } \\
\text { (min.) }\end{array}$} & \multirow[b]{2}{*}{$\%$} & \multicolumn{2}{|l|}{ Gravação } & \multicolumn{2}{|c|}{ Desenvolvimento } & \multirow{2}{*}{$\begin{array}{c}\text { Extrusão } \\
\text { (min.) }\end{array}$} & \multicolumn{2}{|c|}{ PCP } & \multirow[b]{2}{*}{$\%$} \\
\hline & & & $(\min )$. & $\%$ & (min.) & $\%$ & & $\%$ & $(\min )$. & \\
\hline \multicolumn{11}{|l|}{ Janeiro } \\
\hline \multicolumn{11}{|l|}{ Fevereiro } \\
\hline \multicolumn{11}{|l|}{ Março } \\
\hline \multicolumn{11}{|l|}{ Abril } \\
\hline \multicolumn{11}{|l|}{ Maio } \\
\hline Junho & 1.095 & 67 & 200 & 12 & 350 & 21 & 0 & 0 & 0 & 0 \\
\hline Julho & 570 & 25 & 0 & 0 & 1.290 & 58 & 380 & 17 & 0 & 0 \\
\hline \multicolumn{11}{|l|}{ Agosto } \\
\hline \multicolumn{11}{|l|}{ Setembro } \\
\hline \multicolumn{11}{|l|}{ Outubro } \\
\hline \multicolumn{11}{|l|}{ Novembro } \\
\hline Dezembro & & & & & & & & & & \\
\hline
\end{tabular}

Fonte: Dados da pesquisa.

Tabela 6 - Sucata gerada e tempos de máquina operando e parada

\begin{tabular}{|c|c|c|c|c|c|}
\hline Mês & Pedidos & Sucata gerada $(\mathrm{kg})$ & $\begin{array}{l}\text { Máquina parada } \\
\text { (min.) }\end{array}$ & $\begin{array}{l}\text { Máquina parada } \\
(\%)\end{array}$ & $\begin{array}{l}\text { Máquina operando } \\
\text { (min.) }\end{array}$ \\
\hline \multicolumn{6}{|l|}{ Janeiro } \\
\hline \multicolumn{6}{|l|}{ Fevereiro } \\
\hline \multicolumn{6}{|l|}{ Março } \\
\hline \multicolumn{6}{|l|}{ Abril } \\
\hline \multicolumn{6}{|l|}{ Maio } \\
\hline \multirow[t]{2}{*}{ Junho } & 10 & 461 & 1.645 & 2,27 & 72.552 \\
\hline & 11 & 504 & 2.240 & 2,36 & 95.062 \\
\hline \multicolumn{6}{|l|}{ Julho } \\
\hline \multicolumn{6}{|l|}{ Agosto } \\
\hline \multicolumn{6}{|l|}{ Setembro } \\
\hline \multicolumn{6}{|l|}{ Outubro } \\
\hline \multicolumn{6}{|l|}{ Novembro } \\
\hline Dezembro & & & & & \\
\hline
\end{tabular}

Fonte: Dados da pesquisa. 


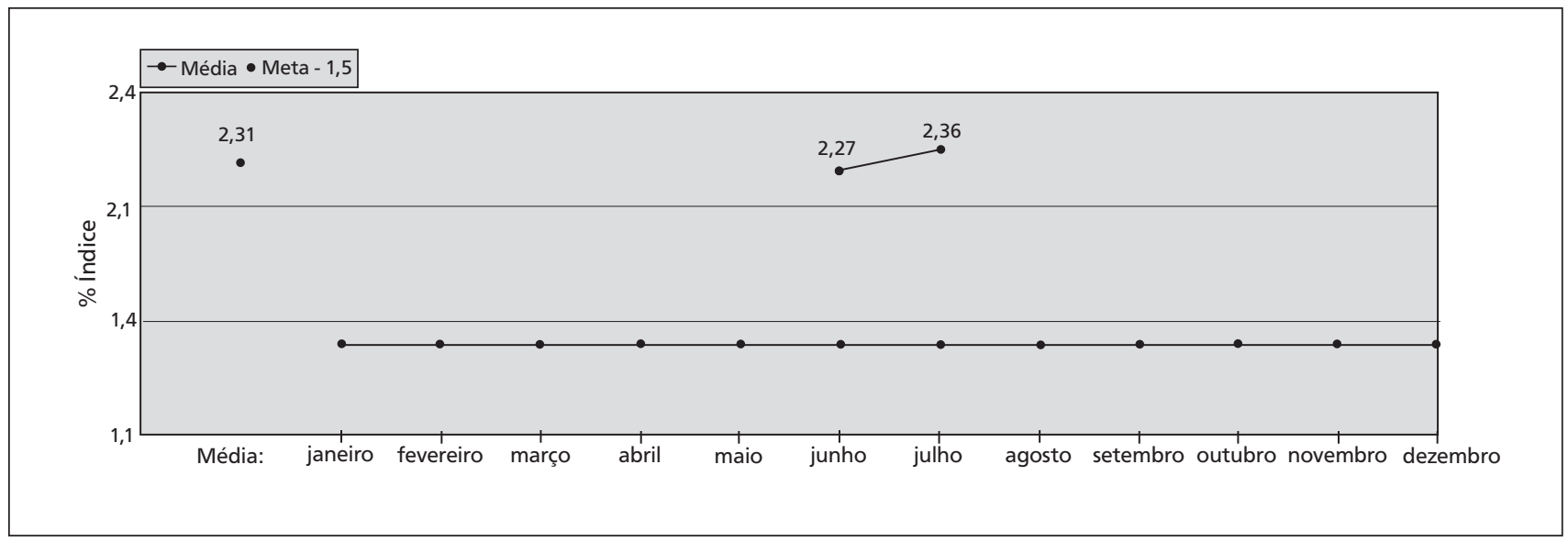

Figura 5 - Tempo de máquina parada e principais causas de problema no setor 2 Fonte: Dados da pesquisa.

Tabela 7 - Principais problemas no setor 3

\begin{tabular}{|c|c|c|c|c|c|c|}
\hline \multicolumn{7}{|c|}{ Análise das aparas $(\mathrm{kg})$} \\
\hline Ocorrências & Maio & $\%$ & Junho & $\%$ & Julho & $\%$ \\
\hline Problema com material (kg) & 210 & 0,06 & 113 & 0,04 & 251 & 0,062 \\
\hline Perda técnica & 3.255 & 1,00 & 2.832 & 0,96 & 3.638 & 0,894 \\
\hline Problema operacional (kg) & 0 & 0,00 & 0 & 0,00 & 23 & 0,006 \\
\hline Problema mecânico $(\mathrm{kg})$ & 39 & 0,01 & 0 & 0,00 & 35 & 0,009 \\
\hline Final de bobina lisa $(\mathrm{kg})$ & 1.644 & 0,51 & 1.940 & 0,66 & 2.062 & 0,507 \\
\hline Problema gerado no setor 2 & 121 & 0,04 & 122 & 0,04 & 40 & 0,01 \\
\hline Sobra de lâmina (cortada - kg) & 5.757 & 1,77 & 4.718 & 1,60 & 9.399 & 2,309 \\
\hline Produção & \multicolumn{2}{|c|}{324.823} & \multicolumn{2}{|c|}{293.982} & \multicolumn{2}{|c|}{407.071} \\
\hline
\end{tabular}

Fonte: Dados da pesquisa.

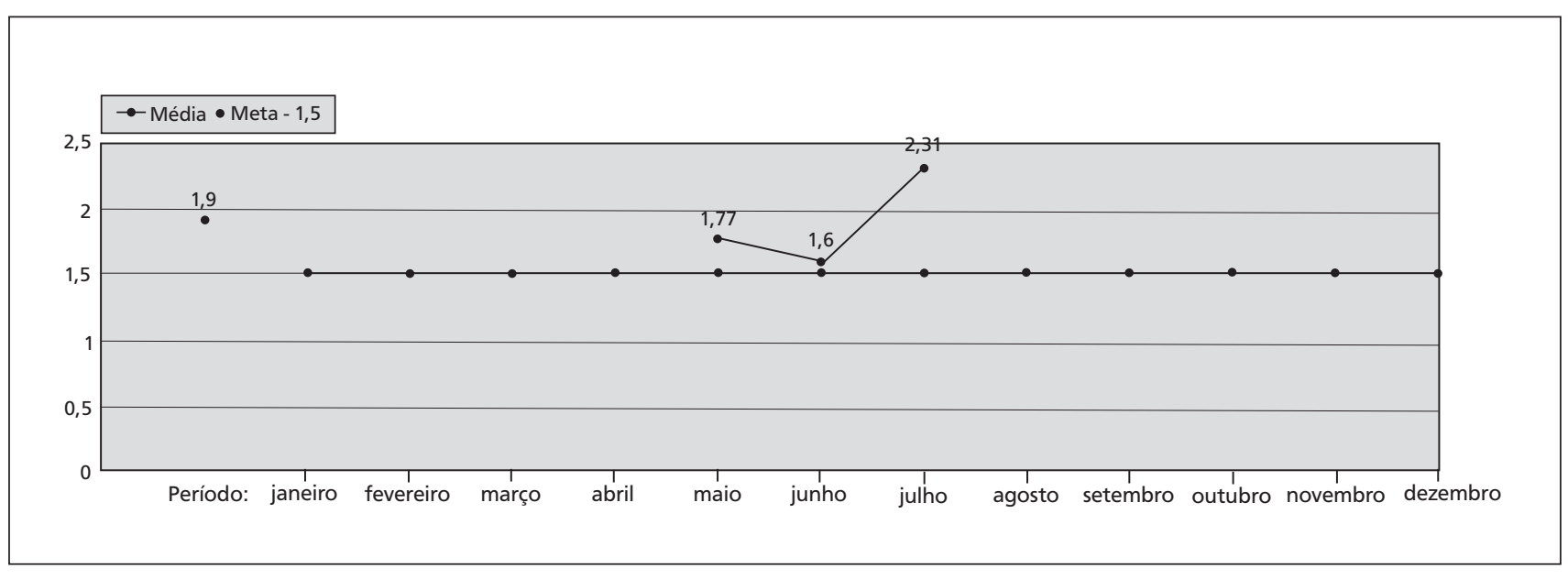

Figura 6 - Indicador de desempenho de sobra de lâmina plástica cortada no setor 3 Fonte: Dados da pesquisa. 
Observando-se o gráfico da Figura 6, percebe-se que também este indicador está fora de controle, causando perdas diversas para a empresa, tanto em produtividade quanto na qualidade de seus produtos. Já para o setor 4 , as causas principais de problema nos três primeiros meses de análise são apresentadas na Tabela 8.

Analisando-se os dados, verifica-se que os maiores índices de apara no setor 4 provêm de problemas gerados pelos setores 2 e 3. Esses índices são mostrados na Figura 7. Não foram consideradas as ocorrências causadas pelo refilo impresso, por esse ser uma perda natural do processo.

A meta para problemas causados pelos outros setores foi estipulada em, no máximo, 0,85\%. 0 gráfico demonstra que essas causas também encontram-se fora de controle, causando diversos problemas de qualidade e paradas de máquina no setor 4. Isso faz com que a empresa perca em competitividade e tenha seus custos aumentados.

0 setor 5 faz a montagem de todos os materiais processados nos demais setores. A partir desse ponto, os produtos são entregues à expedição, prontos para serem enviados aos clientes. Nesse setor, os maiores problemas são relacionados a perdas técnicas de processo. Além disso, também ocorrem perdas causadas pelos setores antecessores do processo produtivo. Esses dados são apresentados na Tabela 9.

Além das perdas técnicas e da apara de corte, observou-se um índice alto de falhas geradas nos setores 2 e 3, que são percebidas no momento de montagem dos produtos. Para controlar estas e passar a identificar suas causas e diminuir este índice, foi tomada como limite $0,85 \%$ de ocorrências geradas em cada um destes setores. 0 acompanhamento para os três primeiros meses é mostrado na Figura 8.

Observando-se os gráficos da Figura 8, vê-se que os índices estão fora de controle, com tendência a aumentar, com índices de 1,3\% para problemas causados no setor 3 e 1,41\% no setor 4 , na média dos três meses analisados.

\section{Conclusões}

Fica evidente a necessidade das organizações controlarem seus processos de produção através de indicadores de desempenho, avaliados através de metas estabelecidas. Pelo estudo de caso realizado, viu-se que a maior parte dos processos e itens avaliados encontrava-se fora de controle.

Tabela 8 - Principais problemas no setor 4

\begin{tabular}{|c|c|c|c|c|c|c|}
\hline \multicolumn{7}{|c|}{ Análise das aparas (kg) } \\
\hline Ocorrências & Maio & $\%$ & Junho & $\%$ & Julho & $\%$ \\
\hline Perda técnica (kg) & 2.065 & 0,57 & 1.446 & 0,50 & 1.412 & 0,417 \\
\hline Problema com material $(\mathrm{kg})$ & 207 & 0,06 & 304 & 0,10 & 390 & 0,115 \\
\hline Problema mecânico (kg) & 7 & 0,00 & 0 & 0,00 & 21 & 0,006 \\
\hline Apara lisa (kg) & 3.020 & 0,83 & 2.260 & 0,77 & 2.045 & 0,604 \\
\hline Problema operacional (kg) & 705 & 0,19 & 1.272 & 0,44 & 891 & 0,263 \\
\hline Refilo impresso (kg) & 7.145 & 1,97 & 6.820 & 2,34 & 8.090 & 2,388 \\
\hline Falha do setor $2(\mathrm{~kg})$ & 3.557 & 0,98 & 3.212 & 1,10 & 5.681 & 1,677 \\
\hline Falha do setor $3(\mathbf{k g})$ & 6.634 & 1,83 & 6.921 & 2,37 & 9.236 & 2,727 \\
\hline Produção (kg) & \multicolumn{2}{|c|}{362.310} & \multicolumn{2}{|c|}{291.615} & \multicolumn{2}{|c|}{338.719} \\
\hline
\end{tabular}

Fonte: Dados da pesquisa. 


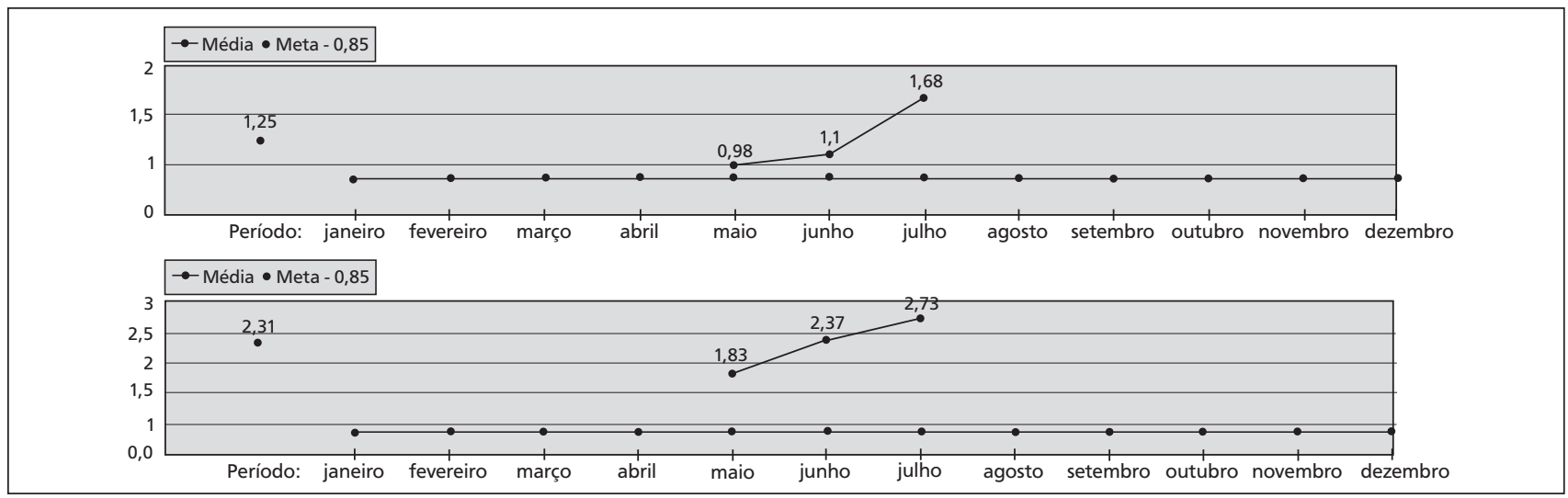

Figura 7 - Indicadores de desempenho por problemas causados nos setores 2 e 3, no setor 4

Fonte: Dados da pesquisa.

Tabela 9 - Principais problemas no setor 5

\begin{tabular}{|c|c|c|c|c|c|c|}
\hline \multicolumn{7}{|c|}{ Análise das aparas (kg) } \\
\hline Ocorrências & Maio & $\%$ & Junho & $\%$ & Julho & $\%$ \\
\hline Apara de corte $(\mathrm{kg})$ & 4.254 & 2,71 & 3.950 & 2,26 & 5.397 & 3,153 \\
\hline Problema com material (kg) & 0 & 0,00 & 0 & 0,00 & 461 & 0,269 \\
\hline Problema mecânico (kg) & 0 & 0,00 & 0 & 0,00 & 0 & 0 \\
\hline Apara lisa (kg) & 581 & 0,37 & 188 & 0,11 & 192 & 0,112 \\
\hline Bobina de acerto $(\mathbf{k g})$ & 286 & 0,18 & 317 & 0,18 & 437 & 0,255 \\
\hline Refilo impresso (kg) & 2.894 & 1,84 & 2.624 & 1,50 & 3.045 & 1,779 \\
\hline Falha geradas no setor $2(\mathbf{k g})$ & 1.630 & 1,04 & 2.249 & 1,29 & 2.711 & 1,584 \\
\hline Falha geradas no setor $3(\mathbf{k g})$ & 1.954 & 1,24 & 1.916 & 1,10 & 3.256 & 1,902 \\
\hline Produção (kg) & \multicolumn{2}{|c|}{157.258} & \multicolumn{2}{|c|}{174.687} & \multicolumn{2}{|c|}{171.154} \\
\hline
\end{tabular}

Fonte: Dados da pesquisa.

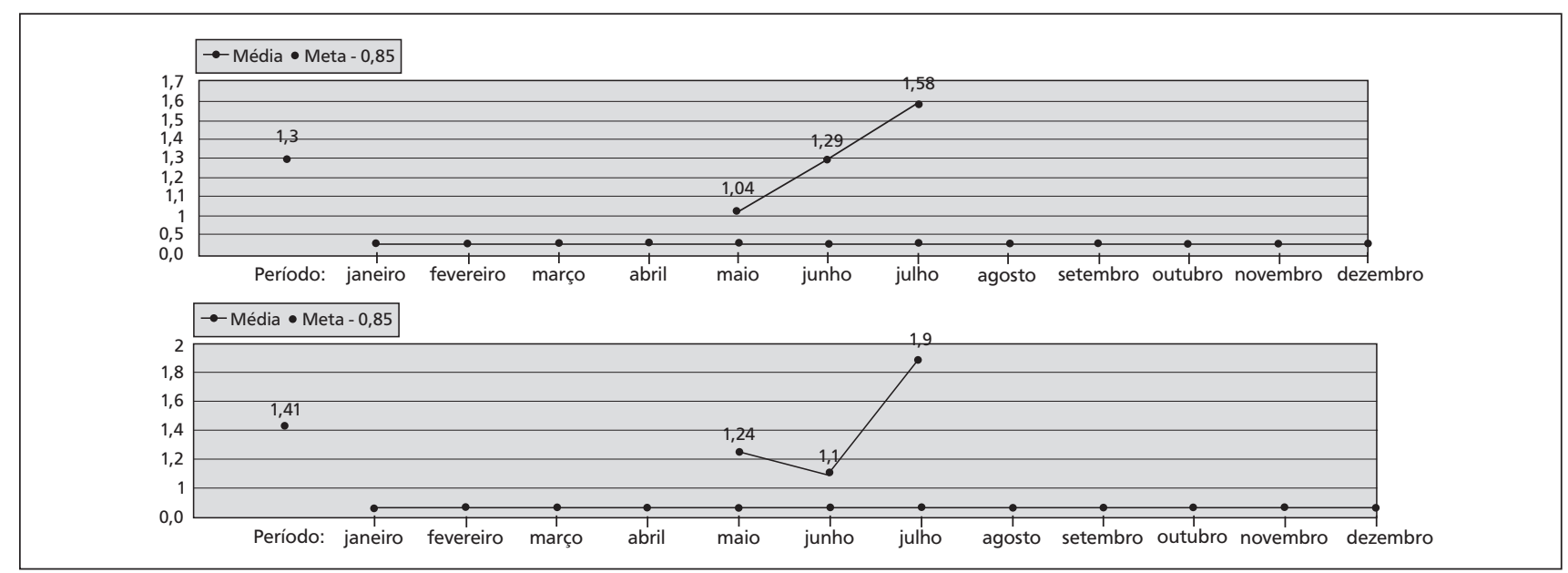

Figura 8 - Indicadores de desempenho por problemas causados nos setores 2 e 3, no setor 5 Fonte: Dados da pesquisa. 
No estudo de caso realizado, percebe-se que apesar de a empresa apresentar resultados financeiros positivos e investir em equipamentos modernos de alta tecnologia e velocidade de produção, a falta de controle dos processos produtivos diminui a competitividade da empresa. A escolha pelo método de medição de desempenho através de indicadores-chave facilitou a coleta de dados e o controle desses, principalmente para uma organização não habituada com a utilização de indicadores para medir o desempenho e na utilização de metas para seus processos. Por isso, os KPI's desenvolvidos por setor foram, inicialmente, voltados para os problemas mais comuns apresentados pelo processo e que interferem diretamente na qualidade e produtividade dos mesmos. A partir desse controle pode-se planejar ações para a eliminação das causas desses processos, buscando a melhoria contínua e a evolução das metas.

Assim, conclui-se que os indicadores de desempenho criados para a empresa estudada tornam-se essenciais para o controle do processo e a tomada de ações de melhoria, o que tornará a empresa mais eficiente e competitiva. Além disso, sugere-se que, para aumentar a competitividade, a empresa busque aplicar a medição do desempenho em outros setores, como: recursos humanos, segurança do trabalho, comercial, financeiro e outros.

\section{Referências}

BROWN, M. G. Winning score: how to design and implement winning scorecards. Portland: Productivity Press, 2000.

CHANG, R. Y.; MORGAN, M. W. Performance scorecards: measuring the right things in the real world. San Francisco: Jossey-Bass, 2000.

CORRÊA, H. L.; HOURNEAUX JUNIOR, F. Sistemas de mensuração e avaliação de desempenho organizacional: estudo de casos no setor químico no Brasil. Contabilidade e Finanças, v. 19, n. 48, p. 50-64, 2008.

CRUZ, S. M. S. et al. Monitoring bioinformatics web services requests and responses through a log based architecture. In: SEMINÁRIO INTEGRADO DE SOFTWARE E HARDWARE, 32., 2005, Campo Grande. Anais... Campo Grande: SEMISH, 2005.

DAVIS, M. M.; AQUILANO, N. J.; CHASE, R. B. Fundamentos da administração da produção. Porto Alegre: Bookman, 2001.
FERREIRA, A. A.; PEREIRA, M. I.; REIS, A. C. F. Gestão empresarial: de Taylor aos nossos dias: evolução e tendências da moderna administração de empresas. São Paulo: Cengage Learning, 2006.

GEORGE, S. 0 sistema Baldrige da qualidade. São Paulo: Makron Books, 1993.

HARRINGTON, H. J. Aperfeiçoando processos empresariais. São Paulo: Makron Books, 1993.

KAPLAN, R. S.; NORTON, D. P. A estratégia em ação. Rio de Janeiro: Campus, 1997.

KAPLAN, R. S.; NORTON, D. P. Mapas estratégicos: balanced scorecard. Rio de Janeiro: Campus, 2004.

LATORRE, V.; ROBERTS, M.; RILEY, M. J. Development of a systems dynamics framework for KPIs to assist project managers' decision making processes. Revista de la Construcción, v. 9, n. 1, p. 39-49, 2010.

LOZECKY, J. Mensuração do resultado. Revista Contemporânea de Contabilidade, v. 1, n. 1, p. 97-114, 2004.

NATIONAL INSTITUTE OF STANDARDS AND TECHNOLOGY NIST. 2011 - 2012 Criteria for performance excellence. Gaitersburg: National Institute of Standards and Technology, 2011.

NEELY, A.; ADAMS, C.; KENNERLEY, M. The performance prism: the scorecard for measuring and managing business success. London: Prentice Hall, 2002.

OLIVEIRA, K. F. de et al. Processo de medição de desempenho em micro e pequenas empresas de um arranjo produtivo local. INGEPRO Inovação, Gestão e Produção, v. 2, n. 11, p. $48-59,2010$.

RENTES, A. F. TransMeth: proposta de uma metodologia para condução de processos de transformação de empresas. 2000. 229 f. Tese. (Doutorado em Engenharia) Universidade de São Paulo, São Paulo, 2000.

Recebido: 26/09/2011

Received: 09/26/2011

Aprovado: $11 / 10 / 2011$

Approved: $10 / 11 / 2011$ 